\title{
Effects of postselected von Neumann measurement on nonclassicality of single-photon- added coherent state
}

\author{
Yusuf Turek ${ }^{1, *}$ \\ ${ }^{1}$ School of Physics and Electronic Engineering, Xinjiang Normal University, Urumqi, Xinjiang 830054, China
}

(Dated: July 9, 2020)

\begin{abstract}
The effects of von Neumann postselected measurement on nonclassicality of single-photon-added coherent state (SPACS) are studied. Explicit expressions and analytical results for various field properties of SPACS such as the photon number distribution, the Mandel $Q_{m}$ factor and the squeezing parameter of field quadrature after postselected von Neumann measurement are investigated. The results showed that the nonclassicality of SPACS after measurement changed dramatically than initial state. The measurement let SPACS possess more strong sub-Poissonian photon statistics in some definite coupling strength regimes and large weak values which accompanied by low postselection probabilities.
\end{abstract}

PACS numbers: 03.65. Ta, 42.50.-p

Keywords: weak value, postselection, photon statistics, sub-Poissonian states

\section{INTRODUCTION}

The preparation and optimization of non-classical quantum states have great importance in quantum information processing including single photon generation and detection [1], gravitational wave detection [2, 3], quantum teleportation [4-7], quantum computation [8], generation and manipulation of atom-light entanglement [911], and precision measurement [12] etc.. It is well known that the implementation of those processes depends on the generation and optimization of the related input radiation fields such as coherent states [13-15], squeezed states [16-18], even and odd coherent states [19-22], displaced and squeezed number states [23], and binomial states $[24,25]$. Thus, it is worthy to study the inherent properties of radiation fields to find the suitable quantum states for effectively implement the above mentioned quantum information processing. There are some radiation fields which initially have classical properties, but after added some photons possess nonclassical properties. The photon- added coherent states (PACSs) are typical example.

PACSs was introduced by Agarwal and Tara in 1991[26], and this state exhibits an intermediate property between a classical coherent state and a purely quantum Fock state. If we only consider the one photon excitation of a classical coherent field, the generated state is called single-photon-added coherent state (SPACS). SPACS not only have interesting properties, but also be useful for possible future applications including the engineering of quantum states [27] and quantum information protocols [28], entangle state generation [29]. After Zavatta et al first studied the experimental generation of the SPACS and visualized the evolution of the quantum-to-classical transition [30], various schemes to generation [31, 32] and enhance the nonclassicality of SPACS widely investigated

\footnotetext{
*yusufu1984@hotmail.com
}

[33-35]. In general, the enhancement of nonclassicality of SPACS depends on the optimization of this state, and the optimization of quantum states are related to quantum measurement.

Measurement is a basic concept in physical science and any information of the system can be obtained from the measurement processes. In general, a measurement composed of three parts, i.e., measuring device, measured system and environment. According the requirements of quantum measurement, in a quantum measurement process, there should be have interaction between measuring device (pointer) and measured system and this interaction should be short enough to guarantee the measurement precision. Thus, in quantum measurement theory interaction Hamiltonian can be expressed by von Newmann Hamiltonian as $H=g \hat{A} \otimes \hat{P}[36]$. Here, $\hat{A}$ is the operator of measured system we want to measure, $\hat{P}$ the canonical momentum of measuring device, and $g$ represent the measuring strength. The measurement strength can decide the amount of information of the measured system during after measurement. That is to say, if the interaction strength between the measured system and apparatus (measuring device) is strong, i.e. $g \gg 1$, then we can get the information of the system we want to get by single trial with very small error. Whereas, if the interaction strength between measured system and apparatus is very weak, i.e. $g \ll 1$, the interference between different eigenvalues of the system observable we want to measure still exist and can't distinguish them. In weak coupling case $(g \ll 1)$ we only can get very trivial information of the system by single trial [37]. However, the mission of getting enough information of the system observable in weak coupling regime, introduced a new kind of quantum measurement theory which called weak measurement.

The weak measurement which characterized by postselection and weak value was proposed by Aharonov, Albert, and Vaidman in 1988 [37], and considered as a generalized von Neumann quantum measurement theory. 
In weak measurement theory, the coupling between the pointer and the measured systems is sufficiently weak and the obtained information by single trial is trivial. Even though the postselected weak measurement on single system provides trivial information, by repeating it on an arbitrarily large ensembles of identical system we can determine the average result with arbitrary precision [38] . One of the distinguished properties of weak measurement compared with strong measurement is that its induced weak value of the observable on the measured system can be beyond the usual range of the eigenvalues of that observable [39]. The feature of weak value usually referred to as an amplification effect for weak signals rather than a conventional quantum measurement and used to amplify many weak but useful information in physical systems. So far, the weak measurement technique has been applied in different fields to investigate very tiny effects, such as beam deflection [40-44], frequency shifts [45], angular shifts [46], velocity shifts [47], and even temperature shift [48]. For details about the weak measurement and its applications in signal amplification processes, we refer the reader to the recent overview of the field [49, 50]. In weak measurement we only consider the evolution of unitary operator upto its first order since the interaction strength between the system and measuring device very weak. However, if we want to connect the weak and strong measurement, check to clear the measurement feedback of postselected weak measurement and analyze experimental results obtained in nonideal measurements, the full order evolution of unitary operator is needed [5153], we call this kind of measurement is postselected von Neumann measurement.

The signal amplification properties of weak measurement can be used in state optimization problems. Recently, the state optimization problem by using postselected von Neumann measurement have been presented widely, such as taking the Gaussian states [49, 54], Hermite- Gaussian or Laguerre-Gaussian states, and nonclassical states $[55,56]$. The advantages of non-classical pointer states in increasing postselected measurement precision have been examined in recent studies[56-58]. In Ref. [59], the authors studied the effects of postselected measurement characterized by modular value [60] to show the properties of semi-classical and non-classical pointer states considering the coherent, coherent squeezed, and Schrodinger cat state as a pointer. Most recently, the author of this paper investigated the effects of postselected von Neumann measurement on the properties of singlemode radiation fields [61], and found that postselected von Neumann measurement really changed the photon statistics and squeezing parameters of radiation fields for different weak values and coupling strengths. However, to our knowledge, the effects of postselected von Neumann measurement on the nonclassicality of SPACS has not been previously investigated, neither exactly nor analytically, in any literature.

In this paper, motivated by the previous studies $[58,59,61]$, we investigate the effects of postselected von
Neumann measurement on nonclassicality of SPACS. In order to achieve our goal, we take the spatial degree of freedom of SPACS as pointer and its polarization degree of freedom as measured system. First of all, we obtained the normalized final state of SPACS after postselected von Neumann measurement by taking all interaction strengths between system and measuring device into account. Then calculate the exact expressions and give numerical results of physical quantities of SPACS such as photon number distribution, $Q_{m}$ factor and squeezing parameter. Thu numerical results showed that postselected von Neumann measurement dramatically changed the noncalssicality of SPACS. We notice that in our scheme the measurement strengths and weak values have important role in changing the nonclassicality of SPACS.

The rest of this paper is organized as follows. In Sec. II, we introduce the basic model setup for our scheme. In Sec. III and Sec. IV, we give the details about the effects of postselected von Nuemann measurement on the noncalssicality of SPACS. We calculate the photon number distribution, Mandel $Q_{m}$ factor and squeezing parameter of SPACS, and found that postselected von Nuemann measurement can change the nonclassicality of SPACS by changing the coupling strengths and weak values. We give a conclusion to our paper in Sec. V. Throughout this paper, we use the unit $\hbar=1$.

\section{MODEL SETUP}

In this section, we introduce the related theories to our current research. According to the standard quantum measurement theory, the coupling interaction between system and measuring device is taken to the standard von Neumann type Hamiltonian [36]

$$
H=g \delta\left(t-t_{0}\right) \hat{A} \otimes \hat{P} .
$$

Here, $g$ is a coupling constant and $\hat{P}$ is the conjugate momentum operator to the position operator $\hat{X}$ of the measuring device, i.e., $[\hat{X}, \hat{P}]=i \hat{I} . \quad \hat{A}$ is observable of the measured system we want to measure. In general, to guarantee the measurement precision, the interaction time between measured system and measuring device is too short so that $\int_{0}^{T} g \delta\left(t-t_{0}\right) d t=g$. Thus, the time evolution operator $e^{-i \int_{0}^{T} H d t}$ of our system can be written as $e^{-i g A \otimes \hat{P}}$.

The position operator $\hat{X}$ and momentum operator $\hat{P}$ of measuring device can be written in terms of annihilation (creation) operators, $\hat{a}\left(\hat{a}^{\dagger}\right)$ in Fock space representation as

$$
\begin{aligned}
& \hat{X}=\sigma\left(a^{\dagger}+a\right), \\
& \hat{P}=\frac{i}{2 \sigma}\left(a^{\dagger}-a\right),
\end{aligned}
$$

where $\sigma$ is the width of the beam. We know that the annihilation (creation) operators $a\left(a^{\dagger}\right)$ obey the commutation relation, $\left[a, a^{\dagger}\right]=1$. By substituting Eq.(3) to 
unitary evolution operator $e^{-i g \hat{A} \otimes \hat{P}}$ for system observable $\hat{A}$ which satisfies the property $\hat{A}^{2}=\hat{I}$, we get

$$
e^{-i g \hat{A} \otimes \hat{P}}=\frac{1}{2}(\hat{I}+\hat{A}) \otimes D\left(\frac{s}{2}\right)+\frac{1}{2}(\hat{I}-\hat{A}) \otimes D\left(-\frac{s}{2}\right),
$$

where parameter $s$ defined by $s: \equiv g / \sigma$ and it can characterize the measurement strength of our scheme, and $D(\alpha)$ is a displacement operator with complex $\alpha$ defined by $D(\alpha)=e^{\alpha a^{\dagger}-\alpha^{*} a}$. Note that we can say that the coupling between system and pointer is weak (strong) if $s \ll 1(s \gg 1)$, and in this study we will consider all interaction strengths between system and measuring device.

If we assume that initially the system prepared to $\left|\psi_{i}\right\rangle$ and the initial state of the measuring device is $|\Psi\rangle$, then the evolution of the total system can be written as $e^{-i g \hat{A} \otimes \hat{P}}\left|\psi_{i}\right\rangle|\Psi\rangle$. Since our mission is to study the measurement effects on the properties of state $|\Psi\rangle$, the normalized final state of the measuring device can be obtained by taking the postselection with postselected state $\left|\psi_{f}\right\rangle$ onto $e^{-i g \hat{A} \otimes \hat{P}}\left|\psi_{i}\right\rangle|\Psi\rangle$. After some calculation, it reads

$$
|\Phi\rangle=\beta\left[\left(1+\langle\hat{A}\rangle_{w}\right) D\left(\frac{s}{2}\right)+\left(1-\langle\hat{A}\rangle_{w}\right) D\left(-\frac{s}{2}\right)\right]|\Psi\rangle .
$$

Here, the normalization coefficient $\beta$ defined by

$$
\begin{aligned}
& \beta=\frac{1}{\sqrt{2}}\left[1+\left|\langle\hat{A}\rangle_{w}\right|^{2}+\gamma^{2} e^{-\frac{s^{2}}{2}} \operatorname{Re}\left[\left(1+\langle A\rangle_{w}\right)^{*}\left(1-\langle\hat{A}\rangle_{w}\right) \times\right.\right. \\
& \left.\left.e^{2 \operatorname{siIm}[\alpha]}\left(1+\left(\alpha^{*}+s\right)(\alpha-s)\right)\right]\right]^{-\frac{1}{2}}
\end{aligned}
$$

and

$$
\langle A\rangle_{w}=\frac{\left\langle\psi_{f}|\hat{A}| \psi_{i}\right\rangle}{\left\langle\psi_{f} \mid \psi_{i}\right\rangle}
$$

is the weak value of system observable $A$. In general, the weak value $\langle A\rangle_{w}$ is complex and can beyond the average values of eigenvalues of observable $A$. That is to say, if the preselection state $\left|\psi_{i}\right\rangle$ and postselection state $\left|\psi_{f}\right\rangle$ are almost orthogonal, the $\langle A\rangle_{w}$ can take large values and this feature of weak measurement used as amplification of weak signals of realated physical systems.

In this paper, we consider the spatial transversal freedom of SPACS as measuring device and its polarization of freedom as measured system, and study the effects of the measurement which performed on polarization part on the inherent properties of spatial part of SPACS. Assume that initially we prepared the system to the polarization state $\left|\psi_{i}\right\rangle=\cos \frac{\varphi}{2}|H\rangle+e^{i \delta} \sin \frac{\varphi}{2}|V\rangle$ and consider the observable $\hat{A}=\hat{\sigma}_{x}=|H\rangle\langle V|+| V\rangle\langle H|$. After weak measurement, the system state postselected to $\left|\psi_{f}\right\rangle=|H\rangle$, then the weak value $\left\langle\sigma_{x}\right\rangle_{w}$ can be obtained as

$$
\left\langle\sigma_{x}\right\rangle_{w}=e^{i \delta} \tan \frac{\varphi}{2} .
$$

Here, $|H\rangle$ and $|V\rangle$ are horizontal and vertical polarization of the beam with eigenvalues 1 and -1 , respectively.
From this expression we can see that this weak value is a complex number and its value can be beyond the eigenvalues of $\hat{\sigma}_{x}$. However, this large weak values accompanied by low successful postselection probability, $P_{s}=\left|\left\langle\psi_{f} \mid \psi_{i}\right\rangle\right|^{2}=\cos ^{2} \frac{\varphi}{2}$.

As mentioned earlier, in this paper, we consider SPACS as measuring device, and its expression is given by

$$
|\Psi\rangle=\gamma a^{\dagger}|\alpha\rangle
$$

where $\gamma=\left(1+|\alpha|^{2}\right)^{-1}$ is normalization coefficient, and $|\alpha\rangle=D(\alpha)|\alpha\rangle$ is coherent sate with coherent parameter $\alpha=r e^{i \theta}$. As proposed in original paper of Agarwal [26], this state can be produced in nonlinear processes in cavities, and its first implementation is given with nonlinear optical scheme [30]. It is well known that if we subtract on photon from coherent radiation field, the generated state still is a classical field. i.e., $a|\alpha\rangle=\alpha|\alpha\rangle$. However, a single-photon excitation of a coherent state changes it into something quite different. In other words, the application of the creation operator $a^{\dagger}$ changes completely the classical coherent state into a new quantum state which posses nonclassicality. The purpose of our study is to check the effects of measurement on the nonclassicality of SPACS by taking into account the signal amplification property of postselected von Nuemann measurement.

\section{EFFECTS ON PHOTON STATISTICS}

In this section, in order to investigate the effects of postselected von Nuemann measurement on photon statistics of SPACS, we check the photon number distribution and Mandel $Q_{m}$ factor of SPACS after measurement.

\section{A. Photon number distribution}

In this subsection, we check the effect of postselected von Neumann measurement on photon number distribution of SPACS. The probability of finding $n$ photons under the final state $|\Phi\rangle$ of SPACS after measurement is given by

$$
P(n)=|\langle n \mid \Phi\rangle|^{2} .
$$

The explicit expression of $P(n)$ can be obtained by substituting $|\Phi\rangle$ which is given in Eq. (5) to above expression, and its corresponding numerical results are shown in Fig. 1. From previous studies it can be deduced that the initial SPACS have sub-Poissonian distribution [26], and the solid black curve in Fig. 1(a) represent the photon number distribution of initial SPACS. In Fig. 1 (a) we check the effects of interaction strengths on photon number distribution, the result showed that with increasing the interaction strength, the photon number distribution of SPACS become broader and occur oscillation in definite photon regions. We know that weak value have 

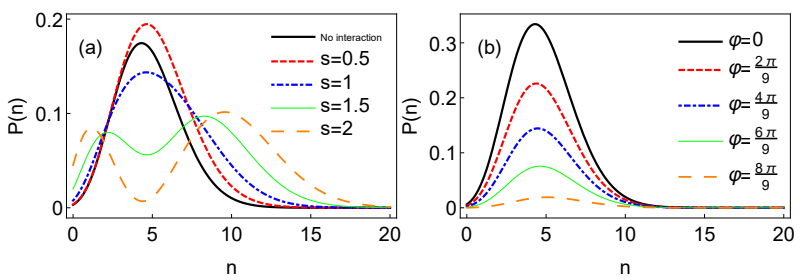

Figure 1. (Color online) Photon number distribution $P(n)$ of SPACS after postselected von Nuemann measurement as a function photon numbers $n$ for different coupling strengths $s$ with fixed weak value $\left(\varphi=\frac{\pi}{3}\right)$ in $(\mathrm{a})$, and for different weak values with fixed coupling strength $(s=0.1)$ in (b). Here, $\delta=\frac{\pi}{4}, r=2, \theta=\frac{\pi}{9}$.

amplification property on weak signals when pre- and pos-selection of system state almost orthogonal. In Fig.1 (b) we display the photon number distribution $P(n)$ of SPACS as a function of photon numbers $n$ for different weak values. As indicated in Fig. 1 (b), in weak measurement regime, the probability of finding $n$ photons decreased as increases the weak value, and photon distribution curves become narrower than initial state. This implies that in weak measurement regime, after postcelect von Neumann measurement, the sub-Poissonian property of SPACS is increased with large weak values.

\section{B. the Mandel $Q_{m}$ factor of SPACS}

The photon variance of initial SPACS less than its mean photon number so that it has nonclassicality which characterized by sub-Poissonian photon statistics [26]. We know that if the underling $P$ - function does not possess or negative Wigner function is negative then the corresponding radiation field have nonclassicality, and SPACS which defined in Eq. (9) possess both of them [26]. However, in this study, in order to check the effects of postselected von Nuemann measurement on nonclassicality of SPACS, we choose the experimentally accessible method-Mandel $Q_{m}$ factor. This parameter is very useful to characterize the nonclassicality of any radiation field and it was introduced by Mandel in 1979 [62]. According to his study, if any distribution which is narrower than Poissonian distribution must correspond to a nonclassical radiation field, and this nonclassicality of that radiation field can be characterized by Mandel $Q_{m}$ factor. The definition of Mandel $Q_{m}$ factor is

$$
Q_{m}=\frac{\left\langle\left(a^{\dagger} a\right)^{2}\right\rangle-\left\langle a^{\dagger} a\right\rangle^{2}-\left\langle a^{\dagger} a\right\rangle}{\left\langle a^{\dagger} a\right\rangle} .
$$

It is clear from the above expression that $Q_{m}=0$ for coherent state and $Q_{m}=-1$ for Fock state. The negativity of $Q_{m}\left(-1 \ll Q_{m}<0\right)$ is a sufficient condition for the field to be nonclassical [63]. However, if $Q_{m}>0$ we can't make a conclusion about the nonclassicality of the field.
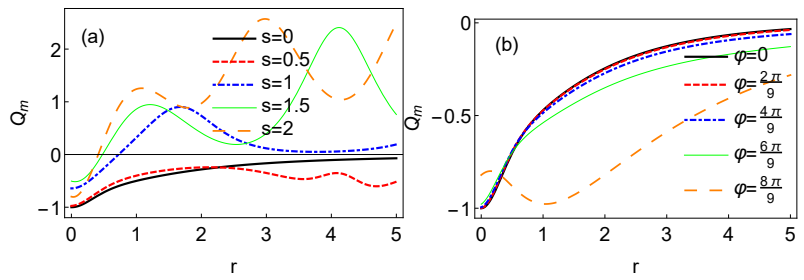

Figure 2. (Color online) The Mandel $Q_{m}$ parameter of SPACS after postselected von Neumann measurement as a function of coherent state parameter $r$ for different coupling strengths $s$ with fixed weak value $\left(\varphi=\frac{\pi}{9}\right)$ in (a), and for different weak values with fixed coupling strength $(s=0.1)$ in $(\mathrm{b})$. Here, $\delta=0, \theta=\frac{\pi}{4}$. by

The $Q_{m}$ parameter for initial pointer state $|\Psi\rangle$ is given

$$
Q_{m, \Psi}=-\gamma^{2} \frac{1+2|\alpha|^{2}+2|\alpha|^{4}}{1+3|\alpha|^{2}+|\alpha|^{4}} .
$$

Furthermore, the $Q_{m, \Phi}$ can be obtained by calculating the expectation values in Eq. (11) under the final normalized state of the pointer $|\Phi\rangle$ after measurement. Since the explicit expression of $Q_{m, \Phi}$ is too cumbersome to show in this paper, we only give its numerical results in Fig. 2. In Fig.2 (a), we plot $Q_{m, \Phi}$ as a function of coherent state parameter $r$, and solid black curve $(s=0)$ represent to initial state case which is described by $Q_{m, \Psi}$. It is showed that the nonclassicality of photon added coherent state is attenuated as coupling strength $s$ increases for definite weak value. In Fig. 2 (b), we check the effects of different weak values on Mandel $Q_{m, \Phi}$ parameter for fixed coupling strength. As indicated in Fig.2 (b), the nonclassicality of photon added coherent state is increased as increases of weak value in weak coupling regime $(s<1)$, and these results keep accordance with numerical results of photon number distribution which presented in Fig. 1. From the Fig. 2 (a) and (b) we can induce that in weak coupling regime the photon statistics of photon added coherent state gradually changed more sub-Poissonian as increases the weak value which accompanied by low postselection probabilities.

\section{EFFECTS ON SQUEEZING PARAMETER OF THE FIELD}

In this section, we study the effects of postselected von neumann measurement on squeezing parameter of SPACS. In general, the squeezing parameter of radiation field is defined as [63]

$$
S_{\phi}=\left(\triangle X_{\phi}\right)^{2}-\frac{1}{2}
$$

where

$$
X_{\phi}=\frac{1}{\sqrt{2}}\left(a e^{-i \phi}+a^{\dagger} e^{i \phi}\right), \quad \phi \in[0,2 \pi]
$$



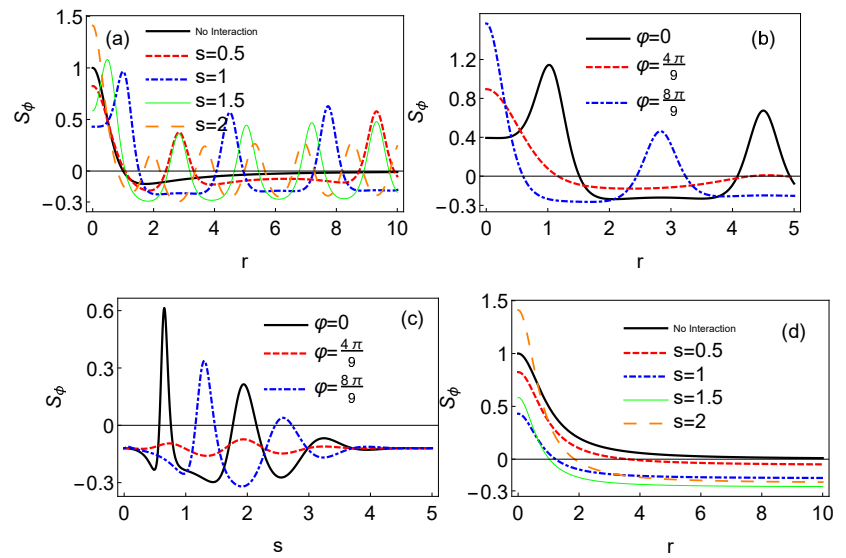

Figure 3. (Color online) The squeezing parameter $S_{\phi}$ of SPACS after postselected von Nuemann measurement. Here, $\delta=0$, and (a) $\varphi=\frac{\pi}{9}, \theta=\frac{\pi}{2}, \phi=\frac{\pi}{2} ;$ (b ) $s=1$, $\theta=\frac{\pi}{2}, \phi=\frac{\pi}{2}$; (c) $r=2, \theta=\frac{\pi}{2}, \phi=\frac{\pi}{2} ;$ (d) $\varphi=\frac{\pi}{9}, \theta=0$, $\phi=\frac{\pi}{2}$.
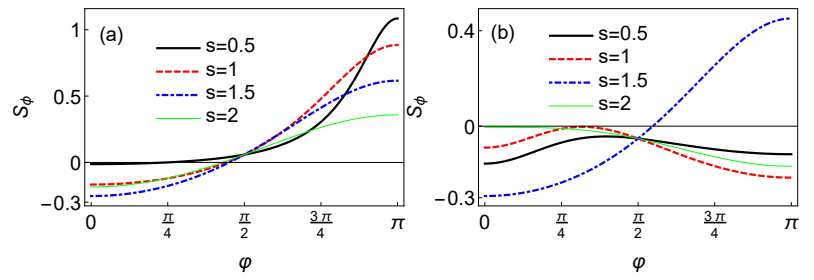

Figure 4. (Color online) The squeezing parameter $S_{\phi}$ SPACS after postselected von Nuemann measurement. (a) $\delta=0$, $r=4, \theta=0, \phi=\frac{\pi}{2}$; (b) $\delta=0, r=4, \theta=\frac{\pi}{2}, \phi=\frac{\pi}{2}$.

is the quadrature operator of the field with

$$
\left[\hat{X}_{\phi}, \hat{X}_{\phi+\frac{\pi}{2}}\right]=i
$$

and

$$
\left(\triangle X_{\phi}\right)^{2}=\left\langle\Phi\left|X_{\phi}^{2}\right| \Phi\right\rangle-\left\langle\Phi\left|X_{\phi}\right| \Phi\right\rangle^{2} .
$$

From the definition of $S_{\phi}$, it can be seen that $S_{\phi} \geq-\frac{1}{2}$. If $-\frac{1}{2} \leq S_{\phi} \leq 0$, then there have squeezing effect of corresponding quadrature of radiation field. For initial pointer state $|\Psi\rangle$, the squeezing parameter $S_{\phi, \Psi}$ is given by

$$
S_{\phi, \Psi}=\gamma^{4}\left[1-|\alpha|^{2} \cos 2(\phi-\theta)\right] .
$$

From this expression, Eq. (17), it can be deduced that only when $|\alpha|^{2}>1$ with $\phi=\theta$, there will occur a squeezing of the corresponding quadrature of SPACS. It is well known that the coherent state is a minimum uncertainty state and squeezing parameter is equal to zero. However, after added on photon, its squeezing feature changed dramatically since the generated new radiation field possess nonclassicality.

The explicit expression of squeezing parameter $S_{\phi}$ of SPACS after postselected von neumann measurement can be calculated by using the final state $|\Phi\rangle$ and Eqs. (13). Since its exact expression is too cumbersome to show here, in this paper we just analyzed the corresponding numerical results. As detailed in Fig. 3 and Fig.4, the postselected von nuemann measurement changed the squeezing effect of photon added coherent state significantly. In Fig. 3(a) we plot the $S_{\phi, \Phi}$ vs coherent state parameter $r$. The black solid curves in Fig. 3 (a) represent the squeezing parameter of initial SPACS $|\Psi\rangle$, i.g. $X_{\phi=\frac{\pi}{2}, \Psi}$ , and other curves represents, $S_{\phi=\frac{\pi}{2}, \Phi}$, after postselected measurement with various coupling coupling strengths for $X_{\phi=\frac{\pi}{2}}$. It can be seen from Fig. 3(a) that squeezing parameter of SPACS is increased in postselected von nuemann measurement for some regions. In Fig. 3(b) and (c) we check the effects of different weak values on squeezing effect of SPACS for fixed coupling strength and fixed coherent state papramter $r$. The results showed that weak values induced by postselected non Nuemann measurement can increase the squeezing effect of SPACS for definite coupling strengths compared to initial SPACS (see the black solid curve in Fig.3(a)). As mentioned above, the squeezing effects of initial SPACS phase dependent and if only the condition $\theta=\phi$ is satisfied then there will occur the squeezing of corresponding quadrature of the SPACS. However, as indicated in Fig. 3(d), the condition $\theta=\phi$ is no more needed to realize the quadrature squeezing of SPACS after postselected non Nuemann measurement. The result indicated that the squeezing effect increased with increasing the coupling strength $s$ between system and measuring device compared to no interaction case (see the black solid curve in Fig. 3 (d)).

In order to further study the effects of weak values on phase catch condition of squeezing parameter of SPACS after poseslected von Nuemann measurement, in Fig. 4 we plot the squeezing parameter $S_{\phi=\frac{\pi}{2}, \Phi}$ for various weak values. It is visible by comparing the Figs. 4(a) and (b) that the condition $\theta=\phi$ is not necessary for realizing the squeezing effect of field quadratures of SPACS after postselected von neumann measurement.

\section{CONCLUSION AND REMARKS}

In this study, we investigated the effects of postselected von Nuemann measurement on nonclassicality of SPACS. In order to achieve our goal, first of all we drived explicit expression of SPACS after measurement by considering all coupling strengths between system and measuring device. We checked the photon number distribution, Mandel $Q_{m}$ factor and squeezing parameter of SPACS after postselected von Nuemann measurement for various system parameters. Our numerical results are given according to the exact expressions of corresponding physical quantities and they showed that postselected von Nuemann measurement can change the nonclassicality which characterized by sub-Poissonian photon distribution of SPACS dramatically. We found that with in- 
creasing the weak values the photon number distribution becomes narrower, the negativity of Mandel $Q_{m}$ factor and squeezing parameter are increased than initial state. Our numerical results also showed that after postselected von Nuemann measurement, the squeezing parameter of SPACS is no more suffer the rigid phase matching condition as initial state.

We anticipate that the presented optimization scheme of nonclassicality of SPACS in this paper would be helpful to provide other effective methods to implement the related practical problems in quantum information processing as listed in introduction part. Furthermore, in this paper we only consider one photon excitation of co- herent radiation field, and it is a simple case of PACSs. Thus, effects of postslected von Nuemann measurement on radiation field properties of more that one photon excitation of coherent radiation field is still worth to study. Work along this line is in progress and results will be presented in near future.

\section{ACKNOWLEDGMENTS}

This work was supported by the National Natural Science Foundation of China (Grant No. 11865017) and the Introduction Program of High Level Talents of Xinjiang Ministry of Science.
[1] G. S. Buller and R. J. Collins, Meas. Sci. Technol 21, 12002 (2010).

[2] H. Grote, M. Weinert, R. X. Adhikari, C. Affeldt, and H. Wittel, Opt. Express 24, 20107 (2016).

[3] F. Y. Khalili, H. Miao, and Y. Chen, Phys. Rev. D 80, 042006 (2009).

[4] S. J. van Enk and O. Hirota, Phys. Rev. A 64, 022313 (2001).

[5] H. Jeong, M. S. Kim, and J. Lee, Phys. Rev. A 64, 052308 (2001).

[6] G. J. Milburn and S. L. Braunstein, Phys. Rev. A 60, 937 (1999).

[7] S. L. Braunstein and H. J. Kimble, Phys. Rev. Lett. 80, 869 (1998).

[8] T. C. Ralph, A. Gilchrist, G. J. Milburn, W. J. Munro, and S. Glancy, Phys. Rev. A 68, 042319 (2003).

[9] L. Li, Y. O. Dudin, and A. Kuzmich, Nature 498, 466 (2013).

[10] B. Hacker, S.Welte, S. Daiss, A. Shaukat, S. Ritter, L. Li, and G. Rempe, Nat. Photon. 13, 110 (2019).

[11] C. A. Muschik, K. Hammerer, E. S. Polzik, and J. I. Cirac, Phys. Rev. A 73, 062329 (2006).

[12] W. J. Munro, K. Nemoto, G. J. Milburn, and S. L. Braunstein, Phys. Rev. A 66, 023819 (2002).

[13] R. J. Glauber, Phys. Rev. 131, 2766 (1963).

[14] U. M. Titulaer and R. J. Glauber, Phys. Rev. 145, 1041 (1966).

[15] D. Stoler, Phys. Rev. D 4, 155 (1971).

[16] Walls and F. D., Nature 306, 141 (1983).

[17] R. Carranza and C. C. Gerry, J. Opt. Soc. Am. B 29, 2581 (2012).

[18] U. L. Andersen, T. Gehring, C. Marquardt, and G. Leuchs, Phys. Scripta. 91, 053001 (2016).

[19] C. Monroe, D. M. Meekhof, B. E. King, and D. J. Wineland, Science 272, 1131 (1996).

[20] A. Ourjoumtsev, H. Jeong, R. Tualle-Brouri, and P. Grangier, Nature 448, 784 (2007).

[21] H. Yuen, Phys. Rev. A 13, 2226 (1976).

[22] V. V. Dodonov, V. I. Mani ${ }^{-}$Ko, and D. E. Nikonov, Phys. Rev. A 51, 3328 (1995).

[23] H. P. Yuen, Phys. Rev. A 13, 2226 (1976).

[24] D. Stoler, B. E. A. Saleh, and M. C. Teich, Opt. Acta 32, 345 (1985).

[25] Lee and T. C., Phys. Rev. A 31, 1213 (1985).
[26] G. S. Agarwal and K. Tara, Phys. Rev. A 43, 492 (1991).

[27] A. P. Lund, H. Jeong, T. C. Ralph, and M. S. Kim, Phys. Rev. A 70, 020101 (2004).

[28] J. Wenger, R. Tualle-Brouri, and P. Grangier, Phys. Rev. Lett. 92, 153601 (2004).

[29] Y. Li, H. Jing, and M.-S. Zhan, J. Phys.B-At Mol Opt. 39, 2107 (2006).

[30] A. Zavatta, S. Viciani, and M. Bellini, Science 306, p.660 (2004).

[31] Y. Li, H. Jing, and M. S. Zhan, Phys. Lett. A 372, 4177 (2008).

[32] M. Barbieri, N. Spagnolo, M. G. Genoni, F. Ferreyrol, R. Blandino, M. G. A. Paris, P. Grangier, and R. TualleBrouri, Phys. Rev. A 82, 063833 (2010).

[33] V. V. Dodonov, M. A. Marchiolli, Y. A. Korennoy, V. I. Mani ${ }^{-}$ko, and Y. A. Moukhin, Phys.Rev.A 58, 4087 (1998).

[34] A. Zavatta, S. Viciani, and M. Bellini, Phys. Rev. A 72, 023820 (2005).

[35] D. Kalamidas, C. C. Gerry, and A. Benmoussa, Phys. Lett. A 372, 1937 (2008).

[36] von Neumann J, Mathematical Foundations of Quantum Mechanics (Princeton University Press, Princeton, NJ, 1955).

[37] Y. Aharonov, D. Z. Albert, and L. Vaidman, Phys. Rev. Lett. 60, 1351 (1988).

[38] J. T. et al., New J. Phys. 12, 013023 (2010).

[39] [39] Y. Aharonov and D. Rohrlich, Quantum ParadoxesQuantum Theory for the Perplexed (Wiley-VCH, Weinheim, 2005).

[40] O. Hosten and P. Kwiat, Science 319, 787 (2008).

[41] L. Zhou, Y. Turek, C. P. Sun, and F. Nori, Phys. Rev. A 88, 053815 (2013).

[42] M. Pfeifer and P. Fischer, Opt. Express 19, 16508 (2011).

[43] P. B. Dixon, D. J. Starling, A. N. Jordan, and J. C. Howell, Phys. Rev. Lett. 102, 173601 (2009).

[44] D. J. Starling, P. B. Dixon, A. N. Jordan, and J. C. Howell, Phys. Rev. A 80, 041803 (2009).

[45] D. J. Starling, P. B. Dixon, A. N. Jordan, and J. C. Howell, Phys. Rev. A 82, 063822 (2010).

[46] O. S. Magaña Loaiza, M. Mirhosseini, B. Rodenburg, and R. W. Boyd, Phys. Rev. Lett. 112, 200401 (2014).

[47] G. I. Viza, J. Martínez-Rincón, G. A. Howland, H. 
Frostig, I. Shomroni, B. Dayan, and J. C. Howell, Opt. Lett. 38, 2949 (2013).

[48] P. Egan and J. A. Stone, Opt. Lett. 37, 4991 (2012).

[49] A. G. Kofman, S. Ashhab, and F. Nori, Phys. Rep. 520, 43 (2012).

[50] J. Dressel, M. Malik, F. M. Miatto, A. N. Jordan, and R. W. Boyd, Rev. Mod. Phys. 86, 307 (2014).

[51] Y. Aharonov and A. Botero, Phys. Rev. A 72, 052111 (2005).

[52] A. Di Lorenzo and J. C. Egues, Phys. Rev. A 77, 042108 (2008).

[53] A. K. Pan and Phys. Rev. A 85, 022122 (2012).

[54] K. Nakamura, A. Nishizawa, and M.-K. Fujimoto, Phys. Rev. A 85, 012113 (2012).

[55] B. de Lima Bernardo, S. Azevedo, and A. Rosas,
Opt. Commun. 331, 194 (2014).

[56] Y. Turek, H. Kobayashi, T. Akutsu, C.-P. Sun, and Y. Shikano, New J. Phys. 17, 083029 (2015).

[57] S. Pang and T. A. Brun, Phys. Rev. Lett. 115, 120401 (2015).

[58] Y. Turek, W. Maimaiti, Y. Shikano, C.-P. Sun, and M. Al-Amri, Phys. Rev. A 92, 022109 (2015).

[59] Y. Turek and T. Eur. Phys. J. D 72, 202 (2018).

[60] Y. Kedem and L. Vaidman, Phys. Rev. Lett. 105, 230401 (2010).

[61] Y. Turek, arXiv:1912.13229 [quant-ph] (2019).

[62] L. Mandel, Opt. Lett. 4, 205 (1979).

[63] G. S. Agarwal, Quantum Optics (Cambridge University Press, Cambridge, England, 2013). 